\title{
Swimming Exercise Ameliorates Symptoms of MOG-Induced Experimental Autoimmune Encephalomyelitis by Inhibiting Inflammation and Demyelination in Rats
}

\author{
Ji-Youn Kim ${ }^{1}$, Eun-Surk Yi ${ }^{1}$, Hyunju Lee ${ }^{2}$, Jun-Su Kim³ ${ }^{3}$, Yong-Seok Jee ${ }^{4}$, Sung-Eun Kim ${ }^{5}$, Chang-Ju Kim ${ }^{5}$, Il-Gyu Ko ${ }^{5}$ \\ ${ }^{1}$ Department of Exercise Rehabilitation \& Welfare, Gachon University, Incheon, Korea \\ ${ }^{2}$ Department of Health Administration, College of Medical Health, Jungwon University, Goesan, Korea \\ ${ }^{3}$ Department of Sports \& Outdoors, College of Health Industry, Eulji University, Seongnam, Korea \\ ${ }^{4}$ Research Institute of Sports and Industry Science, Hanseo University, Seosan, Korea \\ ${ }^{5}$ Department of Physiology, College of Medicine, Kyung Hee University, Seoul, Korea
}

Purpose: Multiple sclerosis is an autoimmune disease that affects the central nerve system, resulting in cumulative loss of motor function. Multiple sclerosis is induced through multiple mechanisms and is caused by inflammation and demyelination. This study aims to evaluate the neuroprotective effect of swimming exercise in experimental autoimmune encephalomyelitis (EAE) rats, an animal model of multiple sclerosis.

Methods: EAE was induced by an intradermal injection of 50- $\mu$ g purified myelin oligodendrocyte glycoprotein 33-55 $\left(\mathrm{MOG}_{33-55}\right)$ dissolved in 200- $\mu \mathrm{L}$ saline at the base of the tail. The rats in the swimming exercise group were made to swim for 30 minutes once pert a day for 26 consecutive days, starting 5 days after induction of EAE. To compare the effect of swimming exercise with interferon- $\beta$, a drug for multiple sclerosis, interferon- $\beta$ was injected intraperitoneally into rats of the EAE-induced and interferon- $\beta$-treated group during the exercise period.

Results: Injection of $\mathrm{MOG}_{33-55}$ caused weight loss, decreased clinical disability score, and increased level of pro-inflammatory cytokines and inflammatory mediators in the lumbar spinal cord. Loss of motor function and weakness increased demyelination score. Swimming exercise suppressed demyelination and expression of pro-inflammatory cytokines and inflammatory mediators. These changes promoted recovery of EAE symptoms such as body weight loss, motor dysfunction, and weakness. Swimming exercise caused the same level of improvement as interferon- $\beta$ treatment.

Conclusions: The results of this experiment suggest the possibility of swimming exercise in urological diseases that are difficult to treat. Swimming exercises can be considered for relief of symptom in incurable multiple sclerosis.

Keywords: Multiple sclerosis; Experimental autoimmune encephalomyelitis; Swimming exercise; Inflammation; Demyelination

- Research Ethics: This study was approved by the Institutional Care and use Committee of Kyung Hee University (KHUASP[SE]-18-150).

- Conflict of Interest: No potential conflict of interest relevant to this article was reported.

\section{- HIGHLIGHTS}

- We investigated neuroprotective effect of swimming exercise in EAE rats, an animal model of multiple sclerosis.

- Injection of $\mathrm{MOG}_{33-55}$ caused EAE and showed weight loss, decreased clinical disability score, and increased level of pro-inflammatory cyto-

kines and inflammatory mediators.

- Swimming exercise suppress inflammation and demyelination, resulting in improving motor function in EAE rats.

Corresponding author: Il-Gyu Ko (iD https://orcid.org/0000-0003-2696-6869 Department of Physiology, College of Medicine Kyung Hee University, 26 Kyungheedae-ro, Dongdaemun-gu, Seoul 02447, Korea

E-mail: rhdlfrb@hanmail.net / Tel: +82-2-961-02827 / Fax: +82-2-964-2195

Submitted: March 28, 2020 / Accepted after revision: May 5, 2020 (i) (7) This is an Open Access article distributed under the terms of the Creative Commons Attribution Non-Commercial License (http://creativecommons.org/licenses/by-nc/4.0/) which permits unrestricted non-commercial use, distribution, and reproduction in any medium, provided the original work is properly cited. 


\section{INTRODUCTION}

Multiple sclerosis is an immune-mediated neurological disease of the central nervous system (CNS). Multiple sclerosis causes inflammation, axonal demyelination, and neurodegeneration within the CNS [1]. Depending on the severity and location of the damage in the CNS, it induces symptoms such as muscle cramps and weakness, walking and balance dysfunction, and poor quality of life [2].

The pathophysiological mechanisms of multiple sclerosis are complex, and inflammation and axonal demyelination are likely to play an important pathogenic role. According to previous studies, pro-inflammatory cytokines or inflammatory mediators affect neuronal function, alter synaptic transmission and plasticity $[3,4]$, and promote neuronal damage, negatively affecting the course of multiple sclerosis [5]. In human and animal models of multiple sclerosis, inhibition on pro-inflammatory cytokines such as tumor necrosis factor- $\alpha$ (TNF- $\alpha$ ), interleukin (IL)-1 $\beta$, and IL-6 is associated with reduced severity of the disease $[4,6]$.

Experimental autoimmune encephalomyelitis (EAE) is one of the most commonly used animal models to study multiple sclerosis [7]. EAE is characterized by T cell and monocyte infiltration in the CNS, and this infiltration is manifested by myelinproducing oligodendrocytes, such as proteolipid protein (PLP), myelin basic protein (MBP), and myelin oligodendrocyte glycoprotein (MOG). This inflammatory process causes sequelae such as axonal damage, demyelination, and then progressive hind-limb paralysis [8].

Regular exercise can contribute to suppressing pathogenic infections by enhancing antigen-specific immune responses $[9,10]$. Swimming exercise improves muscle endurance, pro- motes anti-inflammatory action and has a neuroprotective effect $[11,12]$. Compared to running, swimming can improve molecular adaptation, causing more neuroprotective effects [13].

In this study, we evaluated whether swimming exercise affects functional recovery in $\mathrm{MOG}_{33-55}$-induced EAE rats. Clinical disability score was determined and rotarod test, vertical pole test, and grip strength test were performed. We conducted Luxol fast blue staining for the detecting of demyelination and enzyme-linked immunoassay (ELISA) for the measuring of TNF- $\alpha$, IL-1 $\beta$, IL-6 expression. Western blot analysis was performed to detect expression of MBP, PLP, nerve growth factor (NGF), cyclooxygenase-2 (COX-2), and inducible nitric oxide synthases (iNOS).

\section{MATERIALS AND METHODS}

\section{Animals and Grouping}

Forty adult female Lewis rats weighing $210 \pm 5 \mathrm{~g}$ (9 weeks in age) were purchased commercially from breeder (Orient Bio Co., Seongnam, Korea). The rats were randomly divided into 4 groups ( $n=8$ in each group): control group, EAE-induced group, EAE-induced and swimming exercise group, EAE-induced and interferon- $\beta$ (IFN- $\beta$ )-treated group. This experiment was approved by the Institutional Animal Care and Use Committee of Kyung Hee University [KHUASP(SE)-18-150]. All experimental procedures were performed according to the animal care guidelines from the National Institutes of Health and the Korean Institute of Medical Sciences.

\section{EAE Induction and Clinical Disability Score Assessment} The rats were anesthetized by inhalation of sevoflurane (4\%) in oxygen $(30 \%)$ and nitrogen $(70 \%)$ delivered through a face

\section{= Assessments of clinical disability score \& body weight}

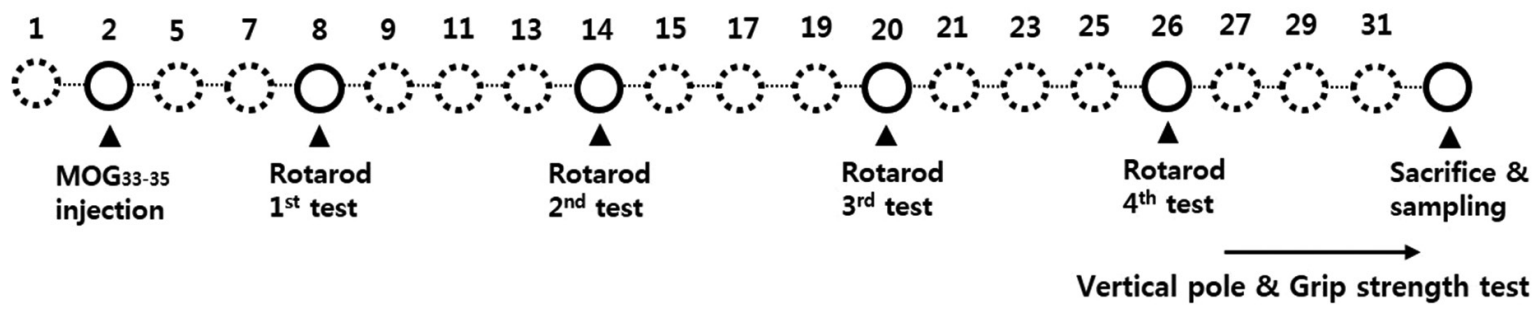

Swimming exercise $\&$ IFN- $\beta$ treatment

Fig. 1. Experimental schedule. MOG, myelin oligodendrocyte glycoprotein; IFN, interferon. 
mask. As described previously [14,15], $50 \mu \mathrm{g}$ of purified MOG 33-55 (MOG $\left.{ }_{33-55}\right)$ was dissolved in 200- $\mu \mathrm{L}$ saline which was homogenized (1:1 v/v) with Freund's complete adjuvant (with mycobacteria). $\mathrm{MOG}_{33-55}$ was injected intradermally through the base of the tail. The animals in the control group received saline injection at similar time. The detailed experimental schedule is presented in Fig. 1.

The body weight and clinical disability score were monitored, once every 2 days for 30 days (14 times, excluding one pretest before EAE induction). The clinical disability score was defined as follows: 0 with no clinical signs, 1 with tail paralysis (or loss of tail tone), 2 with tail paralysis and hind-limb weakness, 3 with hind-limb paralysis, and 4 with complete hindlimb paralysis and front-limb weakness [16].

\section{Swimming Exercise Protocol and Treatment}

The swimming exercise apparatus comprised a plastic tank (90$\mathrm{cm}$ height, 30-cm diameter) filled with water to a depth of 50 $\mathrm{cm}$ at $30^{\circ} \mathrm{C}$. Starting 5 days after EAE induction, the rats in the swimming exercise group were made to swim for 30 minutes, one time a day for 26 consecutive days.

After 2 days of EAE induction, the rats in the IFN- $\beta$ treatment group were injected intraperitoneally with 300,000 units of IFN- $\beta$ (Bayer Schering Pharma, Berlin, Germany) once daily for 26 days (same schedule as the swimming exercise). This IFN- $\beta$ concentration has been demonstrated to be effective as in previous study [17].

\section{Rotarod Test}

According to the previous described method [18], the latency of the rotarod test was used to evaluate motor coordination and balance. The rotarod test was performed 4 times at 6 days intervals after EAE induction. Each rat was placed in a separate compartment on the rotating rod (diameter: $7 \mathrm{~cm}$, fixed velocity: 30 rpm) of the rotarod apparatus (Biological Research Apparatus, Ugo Basile, Varese, Italy). The latency time until fall was automatically recorded by magnetic trip plate. To eliminate stress and fatigue, we limit the latency time for rats to 300 seconds.

\section{Vertical Pole Test}

To measure motor coordination and balance, the vertical pole test was performed according to the previous described method [19]. Each rat was placed face on a pole (3-cm diameter, 150$\mathrm{cm}$ height) covered with cloth tape. Initially, the pole was kept in the horizontal position and then gradually raised to a vertical position. The degree of angle until the rat fell was recorded.

\section{Grip Strength Test}

The forelimb strength was measured by the grip strength test using the grip strength meter 47200 (UgoBasile, Varese, Italy) according to the previous described method [11]. The rat was placed in front of the T-bar to hold the T-bar with the forefoot. We pulled the tail and recorded the force until the rat fell off the T-bar. Peak force was measured in grams. All force measurements were repeated 5 times continuously and the average value was calculated.

\section{Tissue Preparation}

At 31 days after inducing of EAE, the rats were sacrificed after measuring grip strength. The rats were anesthetized with intraperitoneal injection of Zoletil 50 (10 mg/kg; Vibac Laboratories, Carros, France). Then, the rats were transcardially perfused with $50 \mathrm{mM}$ phosphate-buffered saline. The rats were fixed with the freshly prepared solution of $4 \%$ paraformaldehyde in $100 \mathrm{mM}$ phosphate buffer $(\mathrm{pH}, 7.4)$. The lumbar spinal cord was dissected, postfixed with the same fixative overnight, and transferred into 30\% sucrose for cryoprotection. Using a freezing microtome (Leica, Nussloch, Germany), coronal sections of $20-\mu \mathrm{m}$ thickness were cut. An average of 10 lumbar spinal cord sections were obtained from each rat.

\section{Luxol Fast Blue Staining}

Luxol fast blue staining was performed as described previously [20]. Lumbar spinal cord sections of each group were adhered to a gelatin-coated slide. The slides were dehydrated and incubated with $0.1 \%$ Luxol fast blue (Sigma Chemical Co., St. Louis, MO, USA) in $95 \%$ ethanol and glacial acetic acid at $56^{\circ} \mathrm{C}$ overnight. Then, the slides were rinsed with $95 \%$ ethanol and distilled water. The slides were differentiated successively in $0.1 \%$ lithium carbonate and 70\% ethanol. After dehydration and treatment with $100 \%$ xylene, the slides were coverslipped by Permount (Fisher Scientific, Waltham, MA, USA).

Demyelination of the lumbar spinal cord was assessed and we counted score as described previously [21]. Four fields on each slide were analyzed for demyelination of the spinal cord using the Image-Pro plus computer-assisted image analysis system (Media Cybernetics Inc., Silver Spring, MD, USA) attached to a light microscope (Olympus BX61, Tokyo, Japan). The demyelination score was defined as follows: 0 is indicating normal, 1 is indicating one small focal area of demyelination, 2 is 
indicating 2 or 3 areas, 3 is indicating 1 to 2 large areas of demyelination, and 4 is indicating extensive demyelination involving $>20 \%$ of the white matter.

\section{Pro-Inflammatory Cytokines Level}

ELISA was used to detect the concentration of pro-inflammatory cytokines such as TNF- $\alpha$, IL-1 $\beta$, and IL- 6 in lumbar spinal cord tissue. This assay was conducted according to the manufacturer's protocol using an enzyme immunoassay kit (Abcam, Cambridge, UK) for TNF- $\alpha$, IL-1 $\beta$, and IL-6, as described previously [22,23].

\section{Western Blotting}

According to a previous described method [24,25], western blotting was conducted to detect the concentration of NGF, MBP, PLP. The lumbar spinal cord tissues were obtained and immediately frozen at $-80^{\circ} \mathrm{C}$. Then, they were homogenized with lysis buffer containing $10 \%$ glycerol, $1 \%$ Triton X-100, $150 \mathrm{mM} \mathrm{NaCl}, 100 \mathrm{mM}$ NaF, 50mM Tris- $\mathrm{HCl}$ (pH, 8.0), 1.5mM $\mathrm{MgCl}_{2} \cdot 6 \mathrm{H}_{2} \mathrm{O}, 1 \mathrm{mM}$ ethyleneglycoltetra acetic acid, $1 \mathrm{mM}$ phenylmethylsulfonyl fluoride, $1 \mathrm{mM} \mathrm{Na}_{2} \mathrm{VO}_{4}$ and then, were centrifuged for 30 minutes at 14,000 rpm. Bio-Rad colorimetric protein assay kit (Bio-Rad, Hercules, CA, USA) was used to measure content of protein. Protein $30 \mu \mathrm{g}$ was separated on sodium dodecyl sulfate-polyacrylamide gels, and then transferred onto nitrocellulose membrane.

The western blot was initially incubated with following primary antibodies: rabbit MBP antibody, rabbit PLP antibody (1:2,000; Abcam, Cambridge, UK), mouse NGF antibody,

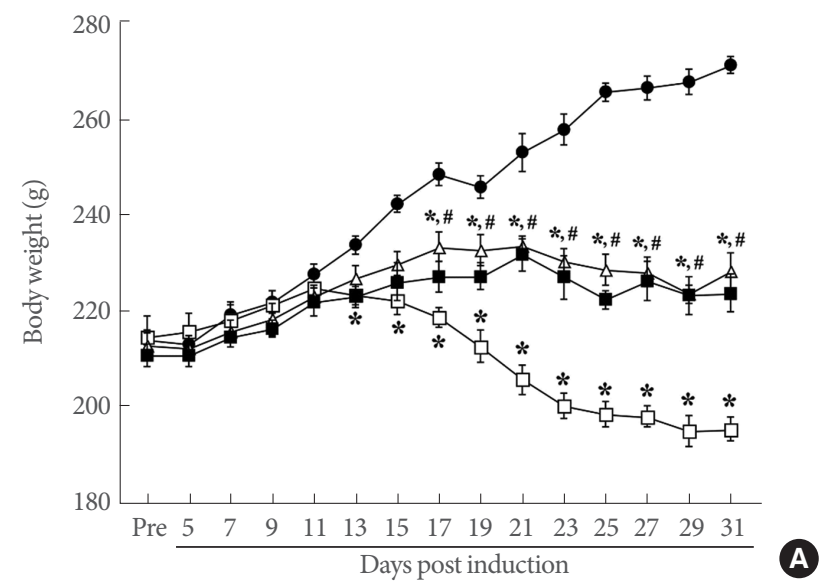

mouse COX-2 antibody, mouse iNOS antibody (1:1,000; Santa Cruz Biotechnology, Santa Cruz, CA, USA), and $\beta$-actin antibody (1:1,000; Santa Cruz Biotechnology). Then, the membrane was treated with following secondary antibodies: horseradish peroxidase-conjugated anti-mouse antibody (1:2,000; Vector Laboratories, Burlingame, CA, USA) for $\beta$-actin, NGF, COX-2, iNOS; and anti-rabbit antibodies (1:3,000; Vector Laboratories) for MBP and PLP.

The step-by-step experiment was conducted under normal laboratory conditions. All steps, except for membrane transfer, were performed at room temperature, which was done at $4^{\circ} \mathrm{C}$ using a cold pack and prechilled buffer. Enhanced chemiluminescence detection kit (Santa Cruz Biotechnology) was used for band detection. In order to compare the relative protein expression of the detected bands, the bands were quantified using a Molecular Analyst version 1.4.1 (Bio-Rad). Control was set to 1.00 for relative quantification.

\section{Statistical Analysis}

Statistical analysis was conducted using one-way analysis of variance and followed by Duncan post hoc test. The results were expressed as mean \pm standard error of the mean. Statistical significance was set at $\mathrm{P}<0.05$.

\section{RESULTS}

\section{Changes in Body Weight and Clinical Disability Score}

The body weight and clinical disability score of $\mathrm{MOG}_{33-35}$-in-

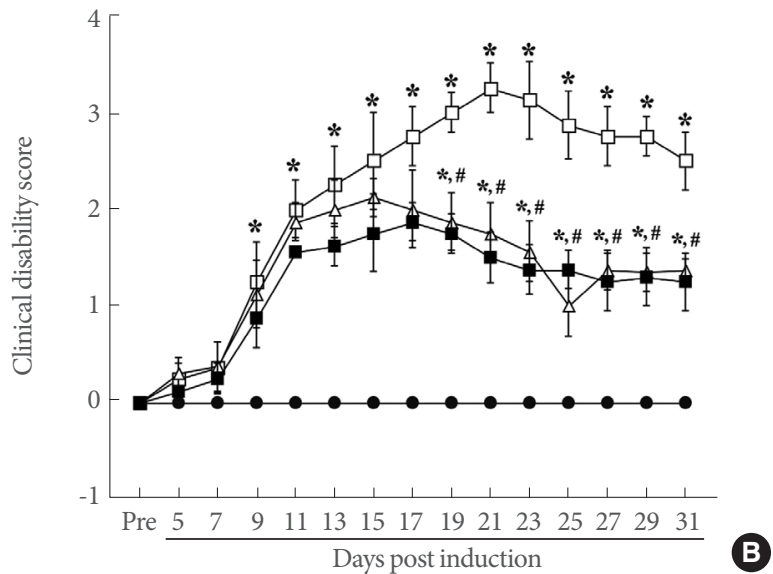

Fig. 2. Body weight and clinical disability score. (A) Body weight during the experiment. (B) Clinical disability score during the experiment. •, control group; $\square$, experimental autoimmune encephalomyelitis (EAE)-induced group; $\triangle$, EAE-induced and swimming exercise group; $\mathrm{n}$, EAE-induced and interferon- $\beta$-treated group. ${ }^{\star} \mathrm{P}<0.05$ compared with the control group. ${ }^{\#} \mathrm{P}<0.05$ compared with the EAE-induced group. 
duced EAE rats are presented in Fig. 2. Tail paralysis or hindlimb weakness was observed after 9 days of EAE induction, and the clinical disability score progressively increased until 21 days after EAE induction $(\mathrm{P}<0.05)$. In addition, compared to the body weight of control rats, there was a sharp decrease in the body weight of EAE-induced rats from the 13th day after EAE induction until the end of the experiment $(\mathrm{P}<0.05)$. This indicates that EAE was well induced in experimental animals, showing tail paralysis, hind-limb weakness, and rapid body weight loss. However, swimming exercise improved EAE symptoms, resulting in a significantly decrease of clinical disability score $(\mathrm{P}<0.05)$. The clinical disability score and body weight of swimming rats were the same as IFN- $\beta$ treated rats.

\section{Changes in Motor Function and Strength}

Motor coordination and balance were assessed with the rotarod and vertical pole tests (Fig. 3A,B). Muscle function was determined with the grip strength test (Fig. 3C). The results indicated that EAE induction negatively affected motor coordination and balance $(\mathrm{P}<0.05)$ and a decrease muscle strength $(\mathrm{P}<0.05)$. However, swimming exercise improved motor coordination and balance $(\mathrm{P}<0.05)$ and increased muscle strength $(\mathrm{P}<0.05)$ in EAE rats. The motor function and muscle strength of swimming rats were the same as IFN- $\beta$ treated rats.

\section{Expression of Pro-Inflammatory Cytokines and Inflammatory Mediators}

The concentration levels of pro-inflammatory cytokines (TNF- $\alpha$, IL-1 $\beta$, and IL-6) and inflammatory mediators (COX-2 and iNOS) in the lumbar spinal cord tissue are presented in Fig. 4. The expression of pro-inflammatory cytokines and inflammatory mediators was increased in $\mathrm{MOG}_{33-55}$-induced $\mathrm{EAE}$ rats $(\mathrm{P}<0.05)$. However, swimming exercise inhibited the expression of TNF- $\alpha$, IL-1 $\beta$, IL- 6 , COX-2, and iNOS in the lumbar spinal cord $(\mathrm{P}<0.05)$.

The expression of TNF- $\alpha$, IL-1 $\beta$, IL- 6 , COX-2, and iNOS of swimming rats was the same as IFN- $\beta$ treated rats.

\section{Changes in Demyelination Score and Expression of NGF, MBP, and PLP}

The histological characteristics and demyelination score in $\mathrm{MOG}_{33-55}$-induced EAE rats are presented in Fig. 5A and B. We
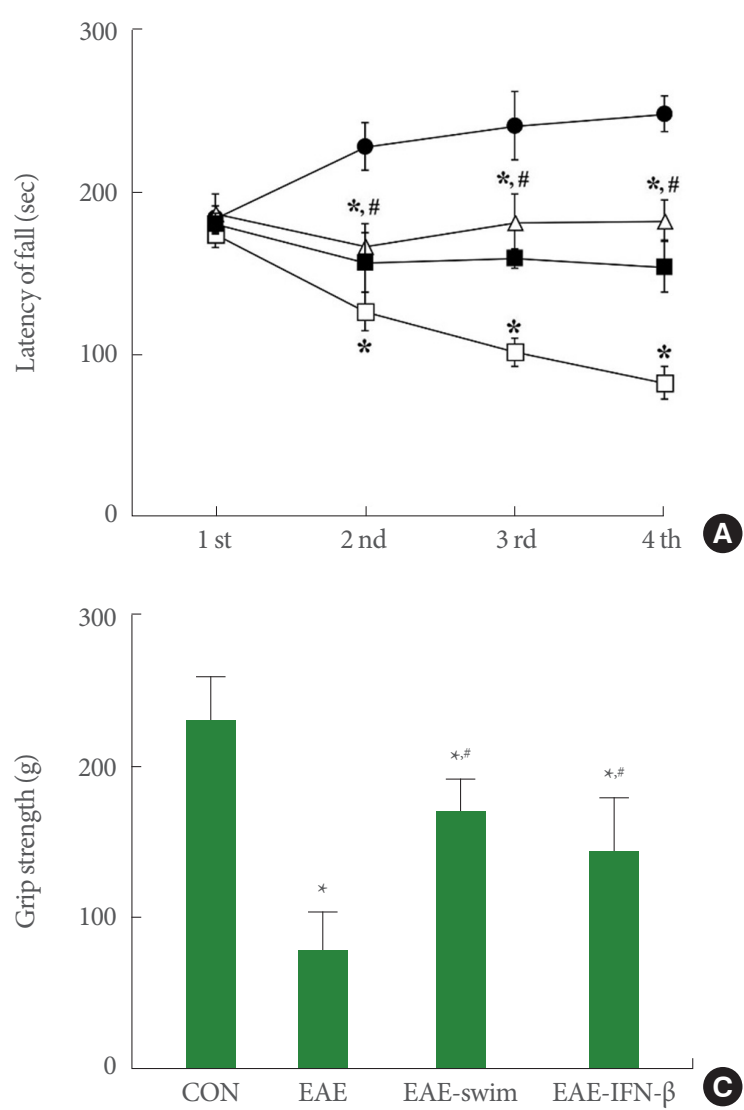

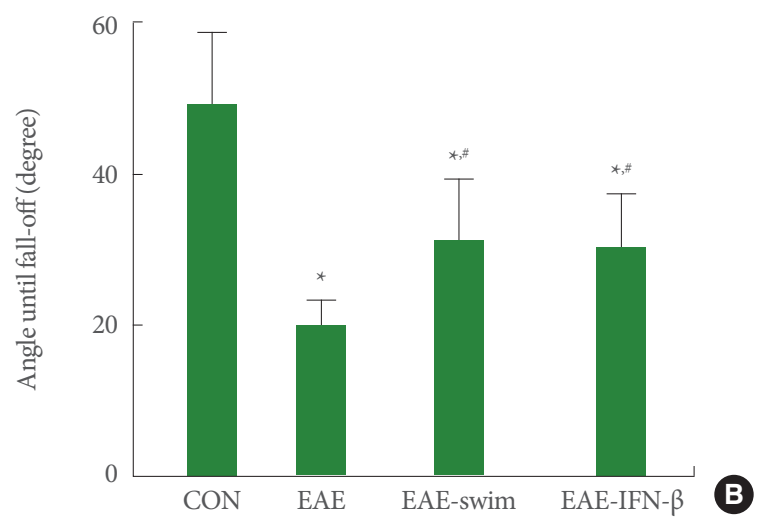

Fig. 3. Motor functions and grip strength. (A) Motor coordination in the rotarod test. $\bullet$, control group; $\square$, experimental autoimmune encephalomyelitis (EAE)-induced group; $\triangle$, EAE-induced and swimming exercise group; $\mathbf{\square}$, EAE-induced and interferon (IFN)- $\beta$-treated group. (B) Balance in the vertical pole test. (C) Muscle strength in the grip strength test. $\mathrm{CON}$, control group; EAE, EAE-induced group; EAE-swim, EAE-induced and swimming exercise group; EAEIFN- $\beta$, EAE-induced and IFN- $\beta$-treated group. ${ }^{\star} \mathrm{P}<0.05$ compared with the control group. ${ }^{*} \mathrm{P}<0.05$ compared with the EAE-induced group. 

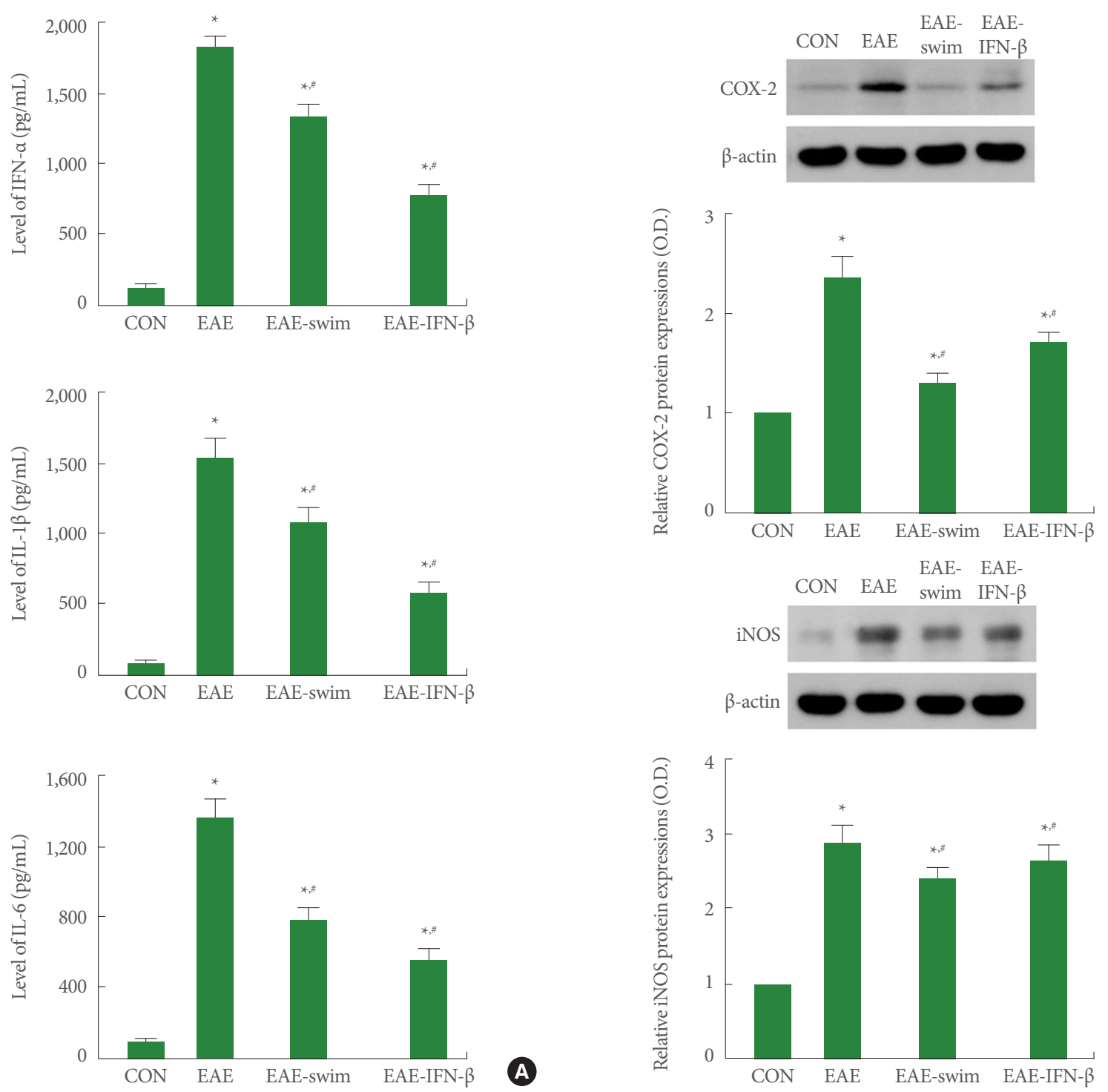

A

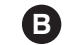

Fig. 4. Expression of pro-inflammatory cytokines and inflammatory mediators. (A) Expression of tumor necrosis factor (TNF)- $\alpha$, interleukin (IL)-1 $\beta$ and IL-6 in the lumbar spinal cord tissue. (B) Expression of cyclooxygenase (COX)-2 and inducible nitric oxide synthase (iNOS) in the lumbar spinal cord tissue. CON, control group; EAE, experimental autoimmune encephalomyelitis (EAE)-induced group; EAE-swim, EAE-induced and swimming exercise group; EAE-IFN- $\beta$, EAE-induced and IFN- $\beta$-treated group. IFN, interferon. ${ }^{\star} \mathrm{P}<0.05$ compared with the control group. ${ }^{*} \mathrm{P}<0.05$ compared with the EAE-induced group.

used western blot analysis to determine the effect of swimming exercise on NGF, MBP, PLP expression (Fig. 5C-E). MOG $33-55$ injection caused demyelination in large areas of the lumbar spinal cord tissue and elevated demyelination score $(\mathrm{P}<0.05)$. Expression of NGF, MBP, and PLP in the lumbar spinal cord was suppressed by induction of EAE $(\mathrm{P}<0.05)$. However, swimming exercise increased expression of NGF, MBP, and PLP, resulting in decreased demyelination score $(\mathrm{P}<0.05)$. Demyelination score and NGF, MBP, and PLP expression of swimming rats was the same as IFN- $\beta$ treated rats.

\section{DISCUSSION}

MOG $_{33-55}$ peptide is an important pathogenic component contributing to EAE induction. The EAE rat model of multiple sclerosis is a representative model of multiple sclerosis because this model markedly demonstrates hind-limb paralysis, motor

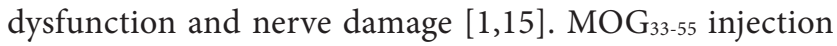



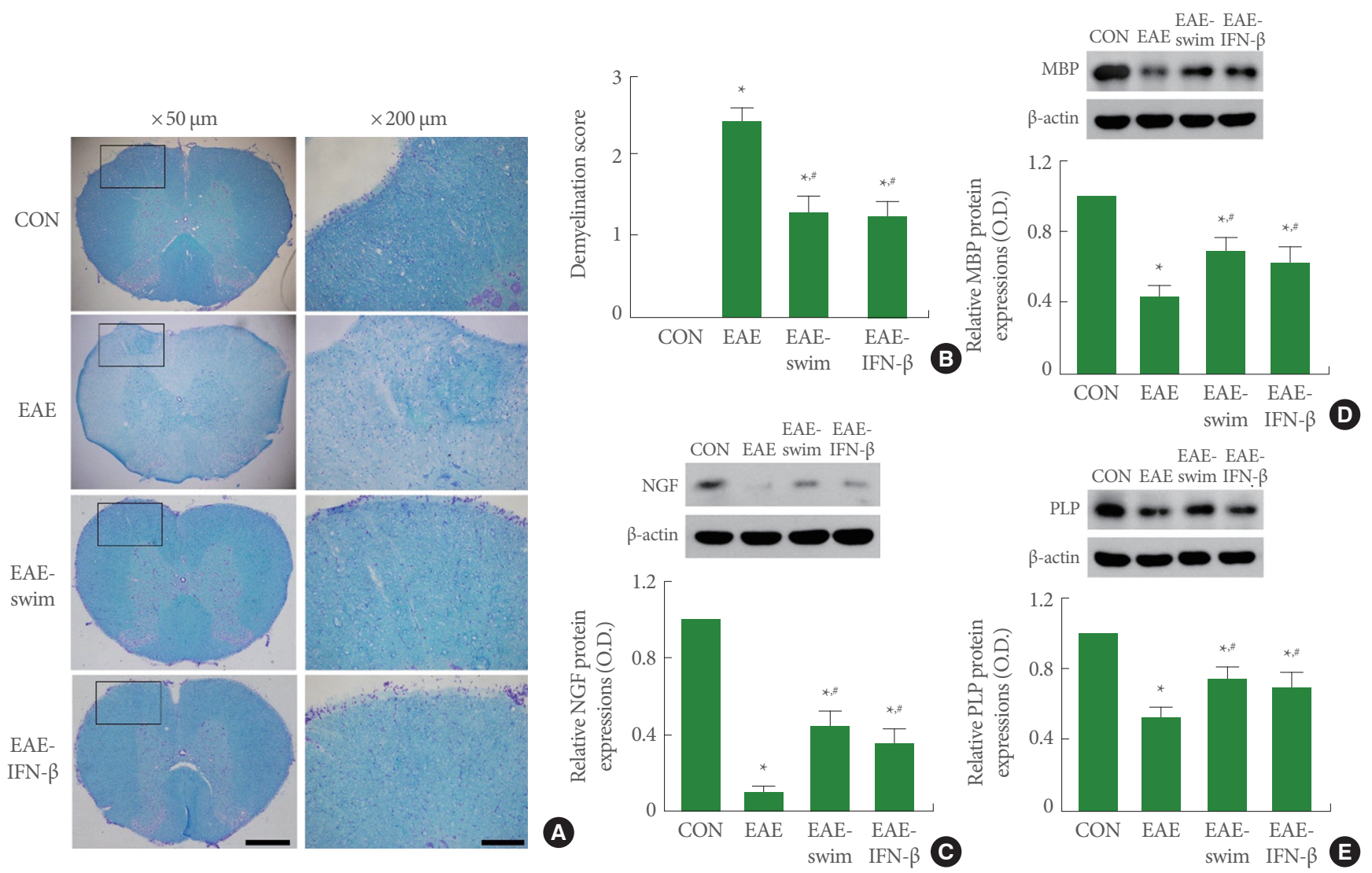

Fig. 5. Demyelination score and expression of nerve growth factor (NGF), myelin basic protein (MBP), and proteolipid protein (PLP). (A) Expression of demyelination in the lumbar spinal cord tissue. $\square$ means demyelination evaluation site. The scale bar represents $50 \mu \mathrm{m}$ (left) and $200 \mu \mathrm{m}$ (right). (B) Demyelination score in each group. (C) Expression of NGF in the lumbar spinal cord tissue. (D) Expression of MBP in the lumbar spinal cord tissue. (E) Expression of PLP in the lumbar spinal cord tissue. CON, control group; EAE, experimental autoimmune encephalomyelitis (EAE)-induced group; EAE-swim, EAE-induced and swimming exercise group; EAE-IFN- $\beta$, EAE-induced and IFN- $\beta$-treated group. ${ }^{\star} \mathrm{P}<0.05$ compared with the control group. ${ }^{*} \mathrm{P}<0.05$ compared with the EAE-induced group.

caused motor dysfunction by secreting pro-inflammatory cytokines and inflammatory exudates [7,21]. Intradermal $\mathrm{MOG}_{33-55}$ injection in our rats indicated weight loss and increased clinical disability score, thus reducing motor coordination, balance, and grip strength. Our study showed the same results as previous study showing increased clinical disability scores and motor dysfunction by $\mathrm{MOG}_{33-55}[3,14]$.

EAE induction enhances axonal damage through increase levels of pro-inflammatory cytokines and inflammatory exudates $[4,26,27]$. In the present study, increased production of pro-inflammatory cytokines (TNF- $\alpha$, IL-1 $\beta$, and IL- 6 ) and inflammatory mediators (COX-2 and iNOS) in the lumbar spinal cord site may be attributed to EAE induction. The present results showed that symptoms of EAE worsen by overproduction of pro-inflammatory cytokines and inflammatory mediators.
Regular physical exercise reduces neuronal degeneration and inflammation [25,28]. In particular, acute or chronic physical exercise may further affect the number and function of diverse cells of innate immune system, including neutrophils, monocytes and natural killer cells $[29,30]$. There is additional evidence that increase in lymphocyte occurs during and after exercise, affects both $\mathrm{T}$ - and $\mathrm{B}$ cells, and regulates balance of proinflammatory and anti-inflammatory cytokines $[10,29]$. In the present results, we found that swimming exercise significantly inhibited the production of pro-inflammatory cytokines and inflammatory mediators to the same extent as IFN- $\beta$ treatment. Swimming exercise promoted mononuclear cell infiltrating and reduction in pro-inflammatory environment 10 days after the induction of EAE, attenuating the severity EAE symptoms in rodents [12]. The results of our experiments showed that swim- 
ming exercise inhibits the production of pro-inflammatory and anti-inflammatory cytokines.

Increased MBP autocatalysis in the brain of multiple sclerosis may contribute to the production of immune dominant epitopes. A decrease in MBP indicates demyelinating status in multiple sclerosis [31]. Likewise, PLP plays a role in the compaction, stabilization, and maintenance of myelin sheaths, as well as in oligodendrocyte development and axonal survival, hence, reduced PLP in EAE indicates demyelination [30]. NGF can contribute to CNS health through myelin biosynthesis [14]. Increased brain NGF may help prevent nerve loss and weaken disease progression in EAE rodents [32]. In the present results, the expression of MBP, PLP, and NGF was decreased after $\mathrm{MOG}_{33-55}$ injection, suggesting that induction of EAE initiated demyelination and neuronal loss. Eventually, reduction in MBP, PLP, and NGF expression increased demyelination scores in the lumbar spinal cord region.

Physical exercise has the effect of regulating immunity by balancing $\mathrm{T}$ helper cell-1 and T helper cell-2 [33,34], thus reducing the demyelination and inflammatory response in EAE. Exercise modulates the components of the immune response, reducing the movement of autoreactive cells across the blood brain barrier into the lumbar spinal cord $[34,35]$. Presently, we found that expression of MBP, PLP, and NGF was increased by swimming exercise in $\mathrm{MOG}_{33-55}$-induced $\mathrm{EAE}$ rats. In addition, swimming exercise reduced the demyelination score in the lumbar spinal cord region. The present results indicated that swimming exercise improves clinical symptoms by reducing immune cell infiltration and preserving axons in the lumbar spine of EAE rats.

Our results show that swimming exercise inhibits inflammation and demyelination and improves motor function and clinical impairment in $\mathrm{MOG}_{33-55}$-induced EAE rats. In addition, regular swimming caused the same level of improvement as IFN- $\beta$ treatment. The results of this experiment suggest the possibility of improving the symptoms of swimming exercise in urological diseases that are difficult to treat. Thus, swimming exercises can be considered for symptom relief in incurable multiple sclerosis.

\section{AUTHOR CONTRIBUTION STATEMENT}

\author{
- Conceptualization: JYK, IGK \\ - Formal Analysis: JYK \\ - Investigation: ESY, HL, JSK, YSJ \\ - Methodology: SEK, CJK
}

- Project Administration: IGK

- Writing - Original Draft: IGK

- Writing - Review \& Editing: IGK

\section{REFERENCES}

1. Klaren RE, Stasula U, Steelman AJ, Hernandez J, Pence BD, Woods $J A$, et al. Effects of exercise in a relapsing-remitting model of experimental autoimmune encephalomyelitis. J Neurosci Res 2016;94: 907-14.

2. Lublin FD. Clinical features and diagnosis of multiple sclerosis. Neurol Clin 2005;23:1-15.

3. Centonze D, Muzio L, Rossi S, Cavasinni F, De Chiara V, Bergami $\mathrm{A}$, et al. Inflammation triggers synaptic alteration and degeneration in experimental autoimmune encephalomyelitis. J Neurosci 2009; 29:3442-52.

4. Stampanoni Bassi M, Gentile A, Iezzi E, Zagaglia S, Musella A, Simonelli I, et al. Transient receptor potential vanilloid 1 modulates central inflammation in multiple sclerosis. Front Neurol 2019;10: 30.

5. Rossi S, Motta C, Studer V, Barbieri F, Buttari F, Bergami A, et al. Tumor necrosis factor is elevated in progressive multiple sclerosis and causes excitotoxic neurodegeneration. Mult Scler 2014;20:304-12.

6. Kamińska J, Koper OM, Piechal K, Kemona H. Multiple sclerosis etiology and diagnostic potential. Postepy Hig Med Dosw 2017;71: 551-63.

7. Robinson AP, Harp CT, Noronha A, Miller SD. The experimental autoimmune encephalomyelitis (EAE) model of MS: utility for understanding disease pathophysiology and treatment. Handb Clin Neurol 2014;122:173-89.

8. Kuerten S, Angelov DN. Comparing the CNS morphology and immunobiology of different EAE models in C57BL/6 mice - a step towards understanding the complexity of multiple sclerosis. Ann Anat 2008;190:1-15.

9. Yoon JR, Ha GC, Ko KJ, Kang SJ. Effects of exercise type on estrogen, tumor markers, immune function, antioxidant function, and physical fitness in postmenopausal obese women. J Exerc Rehabil 2018;14:1032-40.

10. Xie Y, Li Z, Wang Y, Xue X, Ma W, Zhang Y, et al. Effects of moderate- versus high- intensity swimming training on inflammatory and CD4+ T cell subset profiles in experimental autoimmune encephalomyelitis mice. J Neuroimmunol 2019;328:60-7.

11. Santana A Junior, Debastiani JC, Kunz RI, Buratti P, Brancalhão RMC, de Fátima Chasko Ribeiro L, et al. Association of sericin and swimming on the phenotype, motor plate, and functionality of the 
denervated plantar muscle of Wistar rats. J Exerc Rehabil 2018; 14:24-31.

12. Bernardes D, Oliveira-Lima OC, da Silva TV, Faraco CCF, Leite HR, Juliano MA, et al. Differential brain and spinal cord cytokine and BDNF levels in experimental autoimmune encephalomyelitis are modulated by prior and regular exercise. J Neuroimmunol 2013;264:24-34.

13. Deforges S, Branchu J, Biondi O, Grondard C, Pariset C, Lécolle S, et al. Motoneuron survival is promoted by specific exercise in a mouse model of amyotrophic lateral sclerosis. J Physiol 2009;587: 3561-72.

14. Patel DI, White LJ. Effect of 10-day forced treadmill training on neurotrophic factors in experimental autoimmune encephalomyelitis. Appl Physiol Nutr Metab 2013;38:194-9.

15. Herold S, Kumar P, Jung K, Graf I, Menkhoff H, Schulz X, et al. CatWalk gait analysis in a rat model of multiple sclerosis. BMC Neurosci 2016;17:78.

16. Bernardes D, Oliveira ALR. Comprehensive catwalk gait analysis in a chronic model of multiple sclerosis subjected to treadmill exercise training. BMC Neurol 2017;17:160.

17. Lubina-Dąbrowska N, Stepień A, Sulkowski G, Dąbrowska-Bouta B, Langfort J, Chalimoniuk M. Effects of IFN- $\beta 1 \mathrm{a}$ and IFN- $\beta 1 \mathrm{~b}$ treatment on the expression of cytokines, inducible NOS (NOS type II), and myelin proteins in animal model of multiple sclerosis. Arch Immunol Ther Exp 2017;65:325-38.

18. Cervantes-Llanos M, Lagumersindez-Denis N, Marín-Prida J, Pavón-Fuentes N, Falcon-Cama V, Piniella-Matamoros B, et al. Beneficial effects of oral administration of C-Phycocyanin and Phycocyanobilin in rodent models of experimental autoimmune encephalomyelitis. Life Sci 2018;194:130-8.

19. Kim JE, Shin MS, Seo TB, Ji ES, Baek SS, Lee SJ, et al. Treadmill exercise ameliorates motor disturbance through inhibition of apoptosis in the cerebellum of valproic acid-induced autistic rat pups. Mol Med Rep 2013;8:327-34.

20. Bhasin M, Wu M, Tsirka SE. Modulation of microglial/macrophage activation by macrophage inhibitory factor (TKP) or tuftsin (TKPR) attenuates the disease course of experimental autoimmune encephalomyelitis. BMC Immunol 2007;8:10.

21. Feng M, Shu Y, Yang Y, Zheng X, Li R, Wang Y, et al. Ulinastatin attenuates experimental autoimmune encephalomyelitis by enhancing anti-inflammatory responses. Neurochem Int 2014;64:64-72.

22. Jeong H, Chung JY, Ko IG, Kim SH, Jin JJ, Hwang L, et al. Effect of polydeoxyribonucleotide on lipopolysaccharide and sevofluraneinduced postoperative cognitive dysfunction in human neuronal SH-SY5Y cells. Int Neurourol J 2019;23(Suppl 2):S93-101.
23. Timóteo RP, Sales-Campos H, Silva MV, da Silva DAA, Da Silva Catarino J, de Sousa MAD, et al. Pemphigus foliaceus patients (Fogo Selvagem) treated with kinesiotherapy presented lower levels of proinflammatory cytokines. J Exerc Rehabil 2019;15:460-7.

24. Kim SE, Ko IG, Ji ES, Jin JJ, Hwang L, Kim SH, et al. Treadmill exercise alleviates circadian rhythm disruption-induced memory deficits by activation of glucocorticoid receptor and brain-derived neurotrophic factor-dependent pathway. Int Neurourol J 2019; 23(Suppl 1):S40-9.

25. Ko IG, Kim CJ, Kim H. Treadmill exercise improves memory by up-regulating dopamine and down-regulating D2 dopamine receptor in traumatic brain injury rats. J Exerc Rehabil 2019;15:504-11.

26. Arnett HA, Wang Y, Matsushima GK, Suzuki K, Ting JP. Functional genomic analysis of remyelination reveals importance of inflammation in oligodendrocyte regeneration. J Neurosci 2003;23:9824-32.

27. Lee MJ, Jang M, Choi J, Lee G, Min HJ, Chung WS, et al. Bee Venom acupuncture alleviates experimental autoimmune encephalomyelitis by upregulating regulatory $\mathrm{T}$ cells and suppressing Th1 and Th17 responses. Mol Neurobiol 2016;53:1419-45.

28. Song SH, Jee YS, Ko IG, Lee SW, Sim YJ, Kim DY, et al. Treadmill exercise and wheel exercise improve motor function by suppressing apoptotic neuronal cell death in brain inflammation rats. J Exerc Rehabil 2018;14:911-19.

29. Walsh NP, Gleeson M, Shephard RJ, Gleeson M, Woods JA, Bishop NC, et al. Position statement. Part one: immune function and exercise. Exerc Immunol Rev 2011;17:6-63.

30. Klaren RE, Motl RW, Woods JA, Miller SD. Effects of exercise in experimental autoimmune encephalomyelitis (an animal model of multiple sclerosis). J Neuroimmunol 2014;274:14-9.

31. Mastronardi FG, Moscarello MA. Molecules affecting myelin stability: a novel hypothesis regarding the pathogenesis of multiple sclerosis. J Neurosci Res 2005;80:301-8.

32. Mattson MP, Scheff SW. Endogenous neuroprotection factors and traumatic brain injury: mechanisms of action and implications for therapy. J Neurotrauma 1994;11:3-33.

33. Arima Y, Harada M, Kamimura D, Park JH, Kawano F, Yull FE, et al. Regional neural activation defines a gateway for autoreactive $\mathrm{T}$ cells to cross the blood-brain barrier. Cell 2012;148:447-57.

34. Pryor WM, Freeman KG, Larson RD, Edwards GL, White LJ. Chronic exercise confers neuroprotection in experimental autoimmune encephalomyelitis. J Neurosci Res 2015;93:697-706.

35. Mifflin KA, Frieser E, Benson C, Baker G, Kerr BJ. Voluntary wheel running differentially affects disease outcomes in male and female mice with experimental autoimmune encephalomyelitis. J Neuroimmunol 2017;305:135-44. 